\title{
Acceptable outcomes with unicompartmental knee replacement and PCL deficiency are achievable: a case series of nine patients
}

\author{
Pengfei $\mathrm{Li}^{1,2,3}$. James Kennedy ${ }^{1}\left[\right.$ · Hasan Raza Mohammad ${ }^{1} \cdot$ Zhihui Pang $^{3} \cdot$ Stephen Mellon ${ }^{1} \cdot$ William Jackson ${ }^{4}$. \\ Andrew Price $^{1,4} \cdot$ Christopher Dodd $^{4}$. David Murray ${ }^{1,4}$
}

Received: 26 March 2020 / Accepted: 11 June 2020 / Published online: 8 July 2020

(c) The Author(s) 2020

\begin{abstract}
Purpose Posterior cruciate ligament (PCL) deficiency is considered to be a contraindication for unicompartmental knee replacement (UKR); however, there is no evidence to support or contradict this. There are occasional circumstances where UKR in PCL deficient patients have been performed where the patient otherwise satisfies the indications for UKR. The aim of this paper is to describe the outcome of UKR in PCL deficient patients.

Methods A retrospective study of patients with painful medial compartment osteoarthritis and PCL deficiency treated with Oxford UKR between 2006 and 2015 was undertaken. Clinical records from a prospectively recorded database were reviewed and outcomes were assessed based on revision rate, Oxford Knee Score (OKS), American Knee Society score and Tegner Activity Score.

Results Nine patients were identified. The median age at surgery was 51 years (range 42-80) and median follow-up was 6 years (range 1-10). There was one bearing dislocation requiring open exchange. The outcome of seven patients was excellent (OKS > 41). Two patients, who were both elderly, had good outcomes (OKS 41 and 39). One patient had a poor outcome, but it is not clear if this was related to the knee as she had a learning disability and examination and radiographs of the knee were satisfactory.

Conclusion The results of this small series suggest that excellent results can be achieved with UKR for selected patients with medial osteoarthritis in a PCL deficient knee that was functioning well before the osteoarthritis developed. On the basis of this a larger study should be undertaken. Until more results are available PCL deficiency should be considered a relative contra-indication to UKR.
\end{abstract}

Level of evidence IV.

Keywords Posterior cruciate ligament $\cdot$ Oxford unicompartmental knee replacement $\cdot$ Functional outcome $\cdot$ Implant survival · Unicondylar knee replacement

This work was completed at the Nuffield Department of Orthopaedics, Rheumatology and Musculoskeletal Sciences (NDORMS), University of Oxford.

James Kennedy

james.kennedy@ndorms.ox.ac.uk

$1 \quad$ Nuffield Department of Orthopaedics, Rheumatology and Musculoskeletal Sciences, University of Oxford, Oxford OX3 7LD, UK

2 Jiangmen Central Hospital/Affiliated Jiangmen Hospital of Sun Yat-sen University, Jiangmen 529030, China

3 Department of Orthopaedics, The First Affiliated Hospital of Guangzhou University of Chinese Medicine, Guangzhou 510405, China

4 Nuffield Orthopaedic Centre, Oxford OX3 7LD, UK

\section{Introduction}

Unicompartmental knee replacement (UKR) is an effective treatment for knee osteoarthritis. Compared to total knee replacement (TKR), UKR has a faster recovery, lower morbidity and mortality, better functional outcomes, and is more cost effective $[1,2,9,19]$. One of the primary aims of UKR is to restore knee kinematics to normal. This is only possible if all the ligaments are functionally intact, even if they are not anatomically normal. The common situation in which there is knee osteoarthritis with functionally normal ligaments is anteromedial osteoarthritis [17]. Therefore the main indication for UKR is anteromedial osteoarthritis with bone-onbone medially, full thickness cartilage laterally and 
functionally normal ligaments. These criteria are satisfied in about half of patients needing knee replacement $[5,16,18]$.

As the ligaments need to be functionally normal to achieve normal kinematics after UKR, posterior cruciate ligament (PCL) deficiency is considered to be a contra-indication [4]. However, this is not evidence based and the role of PCL deficiency in UKR has not previously been studied. Isolated PCL injuries are rare, but after the injury patients often function well until they develop painful medial osteoarthritis. In the author's practice, particularly if there are mitigating circumstance, UKR has occasionally been performed in PCL deficient knees providing the patient otherwise meets the indications for the procedure. The aim of this study was to review the functional and revision outcomes of the few patients with medial osteoarthritis and PCL deficiency who have been treated with UKR. The study hypothesis was that these patients would have an unacceptably high revision rate with poor functional outcomes.

\section{Materials and methods}

A retrospective case series is described of patients with medial osteoarthritis and PCL deficiency who were treated with Oxford UKR by four surgeons. Apart from the PCL deficiency the patients satisfied the indications for UKR, meaning that they had bone-on-bone medial osteoarthritis, functionally intact medial collateral and anterior cruciate ligaments, and full thickness cartilage in the lateral compartment. Age, activity, obesity, chondral ulcers on the medial side of the lateral femoral condyle and the state of the patellofemoral joint (unless there was lateral bone loss, grooving and subluxation) were ignored $[6,7,15]$.

Patients were identified from a prospectively recorded database of UKR, and were included if they had PCL deficiency and a medial UKR (Fig. 1). Patients were excluded if they had other ligament deficiency (e.g., anterior cruciate ligament), osteotomy, or other UKR procedures such as a lateral UKR or patellofemoral replacement. Where PCL deficiency was recorded, the patients' case notes, operation records and clinic letters were reviewed to determine the reason for their PCL deficiency and why they had been offered a UKR. Patients were assessed pre-operatively and at 1,2 and 5 years using a standard protocol of clinical review with functional assessment. Assessments were made by research physiotherapists, independent of the surgical and clinical teams involved in the patients' care. Functional outcomes were assessed using the Oxford Knee Score (with OKS > 41 considered excellent), the American Knee Society objective and functional (AKSS-O and AKSS-F) Score, and the Tegner Activity score. Implant survival was determined with the endpoint being revision, defined as exchange, removal or addition of any component. In addition complications and

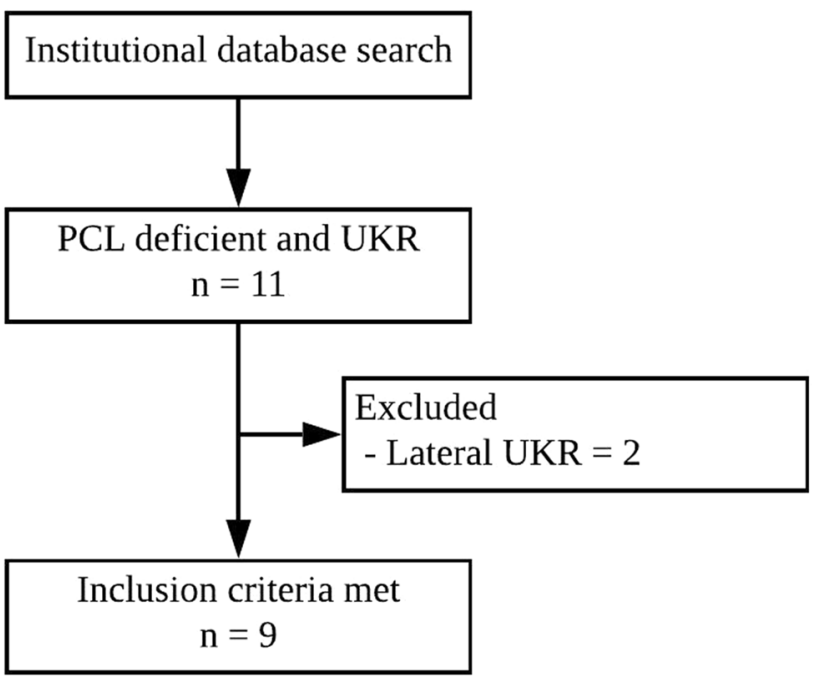

Fig. 1 Patient flow chart. PCL posterior cruciate ligament, UKR unicompartmental knee replacement

re-operations were recorded. In the normal knee at $90^{\circ}$ of flexion, the tibial tuberosity should lie about $1 \mathrm{~cm}$ forward from the distal femoral condyles. The degree of PCL laxity was graded, if done, by using the posterior drawer test intraoperatively. Grade 1 was considered as $>0.5 \mathrm{~cm}$ laxity relative to the contralateral knee, Grade 2 if the anterior tibia could be translated posteriorly to the femoral condyles (indicating $>1 \mathrm{~cm}$ of posterior translation), and in Grade 3 the anterior tibia could be translated posteriorly to the distal femoral condyles [3].

Ethical approval was sought from the local research ethics committee and deemed not required, as information was collected as part of routine patient care.

\section{Results}

Between 2006 and 2015, 9 patients underwent Phase 3 Oxford UKR (Zimmer Biomet, Bridgend, United Kingdom) with a minimally invasive approach for medial osteoarthritis and PCL deficiency. Two were cemented and seven cementless. The median age was 51 (range 42-80) and the median follow-up was 6 years (range 1-10). The outcome of six patients was excellent $(\mathrm{OKS}>41)$, while that of 1 patient was poor (OKS 22). There has been one revision in which a new bearing was inserted for a dislocation, occurring 9 years after the primary surgery. This knee had previously suffered a bearing dislocation that was successfully reduced by a manipulation under anaesthetic at 18 months. There were no serious medical complications but one patient had severe post-operative constipation. The characteristics and outcome scores are shown in Tables 1 and 2. A brief description of each case is given below. 
Table 1 Patient characteristics at surgery

\begin{tabular}{|c|c|c|c|c|c|c|c|}
\hline & Year of surgery & Side & $\begin{array}{l}\text { Age at } \\
\text { surgery } \\
\text { (years) }\end{array}$ & Previous injury & $\begin{array}{l}\text { Cor- } \\
\text { rectable } \\
\text { Varus }\end{array}$ & PCL laxity ${ }^{b}$ & Fixation \\
\hline Patient 1 & 2009 & Left & 43 & Yes & $5^{\circ}$ & Grade 1 & Cementless \\
\hline Patient 2 & 2009 & Right & 43 & Yes & a & a & Cemented \\
\hline Patient 3 & 2010 & Left & 61 & Yes & $\mathrm{a}$ & $\mathrm{a}$ & Cementless \\
\hline Patient 4 & 2010 & Right & 55 & Yes & $\mathrm{a}$ & $\mathrm{a}$ & Cemented \\
\hline Patient 5 & 2011 & Left & 45 & No & $15^{\circ}$ & $\mathrm{a}$ & Cementless \\
\hline Patient 6 & 2011 & Right & 46 & Yes & $\mathrm{a}$ & Grade 2 & Cementless \\
\hline Patient 7 & 2012 & Right & 80 & No & a & Grade 2 & Cementless \\
\hline Patient 8 & 2015 & Right & 51 & Yes & $\mathrm{a}$ & Grade 2 & Cementless \\
\hline Patient 9 & 2015 & Right & 75 & No & $5^{\circ}$ & $\mathrm{a}$ & Cementless \\
\hline
\end{tabular}

$P C L$ posterior cruciate ligament

${ }^{\mathrm{a}}$ Data missing

${ }^{\text {b }}$ Grade 1: $0.5-1 \mathrm{~cm}$ laxity, Grade 2: tibia can be translated to femoral condyles, Grade 3: tibia can be translated beyond the femoral condyles

Table 2 Functional scores and complications

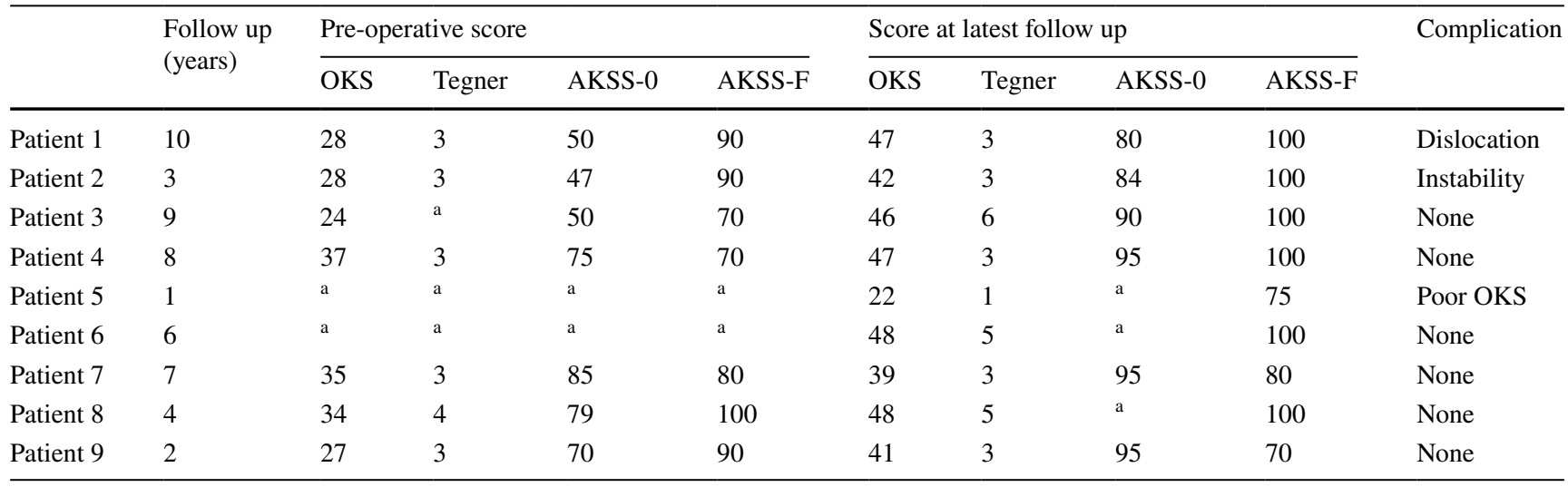

OKS Oxford Knee Score, Tegner Tegner Activity Score, AKSS-O and AKSS-F American Knee Society Score Objective and Functional component respectively

${ }^{\text {a }}$ Data missing

Patient 1 A 43-year-old male who sustained a PCL injury whilst running when 20 years-old, subsequently developed bone-on-bone medial compartment osteoarthritis. 18 months following cementless UKR he sustained a dislocation of the bearing when crouching. He underwent a manipulation under anaesthetic and the bearing was felt to reduce, which was confirmed with fluoroscopy. Eight years later he sustained another dislocation and underwent an open bearing exchange. Whilst the bearing showed signs of anterior wear suggesting anterior impingement, a definite cause could not be found. All the components were found to be well-fixed, and a new $4 \mathrm{~mm}$ bearing was inserted without complication. At 10 years follow-up, the outcome was excellent (OKS 47).

Patient 2 A 43-year-old female who presented with night pain, instability and activity limitation. She had sustained a knee injury in her late 20s whilst playing football, however, it was only at arthroscopic assessment at another centre that she was found to be PCL deficient. Following cemented UKR she was satisfied and pain free on daily activities. However, she continued to have some instability approximately three times a week and anterior knee pain. Despite this, at 3 year follow-up, her OKS was 42 so her outcome was considered excellent.

Patient 3 A 61-year-old male presented with pain and severe medial compartment osteoarthritis. He had sustained a previous PCL injury associated with a tibial fracture. Nine years following the cementless UKR the outcome was excellent (OKS 46).

Patient 4 A 55-year-old male presented with knee pain affecting his golfing, and was found to have severe medial osteoarthritis. When he was young he had sustained an injury to the knee which had caused a PCL disruption and meniscal damage which was treated with an open 
meniscectomy. Eight years following cemented UKR, the outcome was excellent (OKS 47).

Patient 5 A 45-year-old female whose symptoms had deteriorated significantly with pain at night and a dramatically reduced walking distance. She had a learning disability necessitating a residential carer and was overweight. She had a complicated course with severe post-operative constipation. One year following cementless UKR, her OKS was 22, which is categorised as poor. However her range of motion was $0^{\circ}-120^{\circ}$ and the $\mathrm{X}$-ray was satisfactory.

Patient 6 A 46-year-old male who injured his knee when young in a road traffic accident and sustained a PCL injury. He was restricted in his activities by pain. 6 years following the cementless UKR, the outcome was excellent (OKS 48).

Patient 7 An 80-year-old male with PCL deficiency but no definite history of previous major knee trauma presented with significant pain. Seven years following the cementless UKR the outcome was good (OKS 39).

Patient 8 A 51-year-old male presented with pain particularly over the medial aspect of the joint and could not kneel, which was a problem as he was a builder. He had injured his knee skiing 7 years previously. Four years following cementless UKR, the outcome was excellent (OKS 48).

Patient 9 A 75-year-old male presented with pain in his knee such that he could not go up and down stairs normally and walking was limited. X-ray showed severe medial compartment osteoarthritis with bone loss (Fig. 2). He had a significant medical history including pulmonary embolus, coronary artery bypass and spinal problems. He had evidence of PCL deficiency at operation and underwent cementless Oxford UKR (Fig. 3). At 2 year follow-up, the outcome was good, with an OKS of 41.
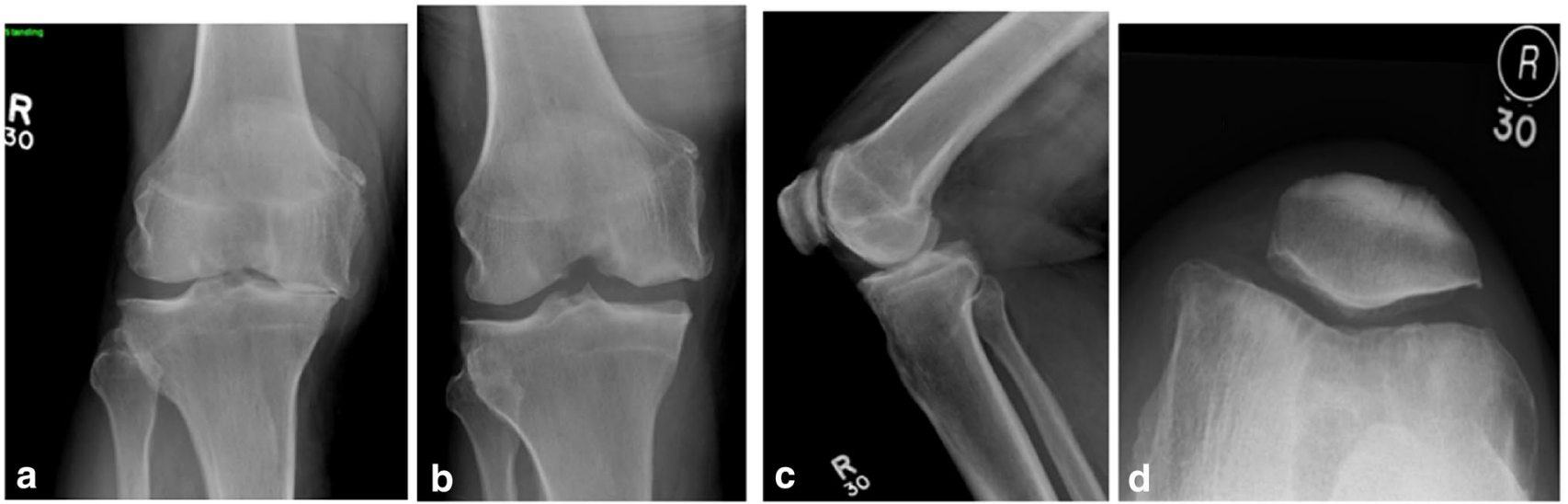

Fig. 2 X-ray showed severe medial compartment arthritis with bone loss. a Rosenberg at $30^{\circ}$ flexion, b Valgus stress, $\mathbf{c}$ lateral, d Skyline 
Fig. 3 X-Ray of same patient as Fig. 2, showing cementless medial UKR in situ
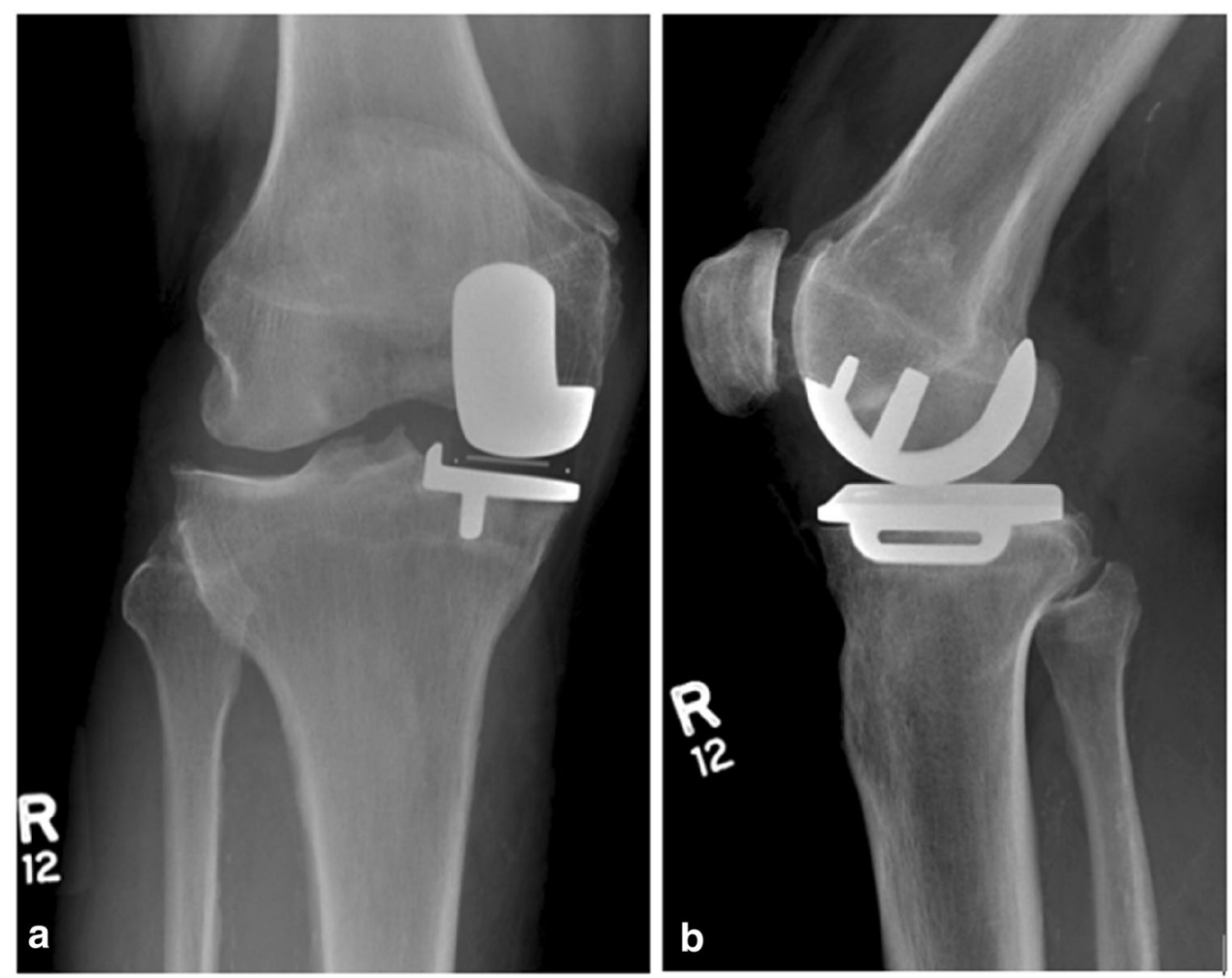

PCL deficiency, who had good function before developing osteoarthritis, as this seems to be a good indicator of their post-operative performance.

The other main subgroup of patients (3/9) that had UKR for medial osteoarthritis with PCL deficiency, were those who it was thought would benefit from the minimally invasive nature of the procedure, which compared to TKR provides a faster recovery with few complications and lower morbidly and mortality [19]. Two of the patients were elderly (age 75 and 80 at the time of surgery) and the younger of these had significant medical co-morbidities. The third patient, although young, was overweight and had a learning disability. The two elderly patients had OKS of 39 and 41 , which are considered good. However the OKS tends to deteriorate with increasing age so scores of this level in these patients who were aged 87 and 77 at follow up should probably be considered to be excellent. The third patient did not do so well but it is not clear if the problems were related to the knee replacement, as clinical examination and radiographs suggested a well-functioning knee. The results would therefore suggest that it is sensible to continue to do UKR with PCL deficiency in the elderly, particularly if they are unfit.

There was only one patient in the intermediate age group, which contains the majority of patients having knee replacement, and presumably the majority of patients with medial osteoarthritis and PCL deficiency needing a knee replacement. This patient, who was aged 61 , had an excellent result
(OKS 46, Tegner 6). As both younger and older patients did well, it would be not unreasonable to do UKR with PCL deficiency in this intermediate group as well, so as to determine how well it performs. These patients should not only benefit from the improved function of the UKR but also the faster recovery and lower morbidity and mortality.

The PCL is the largest and strongest intra-articular knee ligament. It is an essential passive stabiliser of the knee joint, serving as the primary restraint to excessive posterior tibial translation and a significant constraint to internal rotation beyond $90^{\circ}$ of flexion $[8,12]$. However, the PCL is not loaded appreciably during activities of daily living during flexion [11]. PCL injury usually occurs in the context of multiligament knee injuries, but isolated injury does occur and results in altered joint kinematics and can occasionally lead to instability [10]. Following PCL injury, in the medial compartment there is posterior subluxation of the medial tibial plateau with the femoral condyle articulating with the upsloping anterior portion of the medial tibial joint surface which can lead to medial osteoarthritis [10]. Even in young athletes with well-functioning PCL-deficient knees, early medial cartilage degeneration can be detected on MRI [14]. In contrast in the lateral compartment knee kinematics are not altered by PCL rupture so lateral osteoarthritis tends not to occur [10]. These findings help to explain the results of this study. In particular they explain why painful medial osteoarthritis occurs in otherwise well-functioning knees following isolated PCL injury, and why after UKR, which 
treats the pain, good function is likely to occur. They also explain why lateral osteoarthritis is unlikely to occur and hopefully why it will not occur after medial UKR. However, after UKR with PCL deficiency, the kinematics and therefore loading in the medial compartment will be abnormal so the long term outcome remains unknown.

This study is important as it is the first report of the outcome of UKR in PCL deficient knees. The major weakness is the small number of patients. This is because isolated PCL injury is rare and, although PCL deficiency leads to an increased rate of osteoarthritis, the number of PCL deficient patients that could be treated with UKR is very small. In the authors' practice UKR implanted in PCL deficient knees is substantially less than $1 \%$ of all UKR. The other main weakness is the short period of follow up. However, on the basis of the encouraging results in this study, the authors, and, hopefully, other high volume UKR surgeons, will consider doing more cases so hopefully in the future there will be larger studies with longer follow-up on which recommendations can be based. Until then PCL deficiency should be considered to be a relative contra-indication to UKR.

Acknowledgements The authors would like to thank Mrs Barbara Marks and Mrs Jo Brown for their support with this study. HRM would like to thank the University of Oxford for the Henni Mester Scholarship and the Royal College of Surgeons of England Research Fellowship who supported him to undertake research.

\section{Compliance with ethical standards}

Conflict of interest The author or one or more of the authors have received or will receive benefits for personal or professional use from a commercial party related directly or indirectly to the subject of this article. In addition, benefits have been or will be directed to a research fund, foundation, educational institution, or other non- profit organization with which one or more of the authors are associated.

Funding This study did not receive any specific grant from funding agencies in the public, commercial, or not-for-profit sectors. Financial support has been received from Zimmer Biomet.

Ethical approval Ethical approval was sought from the local research ethics committee with formal approval deemed unnecessary under National Health Service research governance arrangements.

Open Access This article is licensed under a Creative Commons Attribution 4.0 International License, which permits use, sharing, adaptation, distribution and reproduction in any medium or format, as long as you give appropriate credit to the original author(s) and the source, provide a link to the Creative Commons licence, and indicate if changes were made. The images or other third party material in this article are included in the article's Creative Commons licence, unless indicated otherwise in a credit line to the material. If material is not included in the article's Creative Commons licence and your intended use is not permitted by statutory regulation or exceeds the permitted use, you will need to obtain permission directly from the copyright holder. To view a copy of this licence, visit http://creativecommons.org/licenses/by/4.0/.

\section{References}

1. Beard DJ, Davies LJ, Cook JA, MacLennan G, Price A, Kent S et al (2019) The clinical and cost-effectiveness of total versus partial knee replacement in patients with medial compartment osteoarthritis (TOPKAT): 5-year outcomes of a randomised controlled trial. Lancet 394:746-756

2. Burn E, Liddle AD, Hamilton TW, Judge A, Pandit HG, Murray DW et al (2018) Cost-effectiveness of unicompartmental compared with total knee replacement: a population-based study using data from the National Joint Registry for England and Wales. BMJ Open 8:e020977

3. Castle JT, Noyes FR, Grood ES (1992) Posterior tibial subluxation of the posterior cruciate-deficient knee. Clin Orthop Relat Res 284:193-202

4. Goodfellow J, O’Connor J, Pandit H, Dodd C, Murray D (2015) Unicompartmental arthroplasty with the Oxford knee. Goodfellow Publishers, Oxford

5. Hamilton T, Pandit H, Lombardi A, Adams J, Oosthuizen C, Clavé A et al (2016) Radiological Decision Aid to determine suitability for medial unicompartmental knee arthroplasty: development and preliminary validation. Bone Jt J 98:3-10

6. Hamilton T, Pandit H, Maurer D, Ostlere S, Jenkins C, Mellon S et al (2017) Anterior knee pain and evidence of osteoarthritis of the patellofemoral joint should not be considered contraindications to mobile-bearing unicompartmental knee arthroplasty: a 15-year follow-up. Bone Jt J 99:632-639

7. Kendrick B, Rout R, Bottomley N, Pandit H, Gill H, Price A et al (2010) The implications of damage to the lateral femoral condyle on medial unicompartmental knee replacement. J Bone Jt Surg Br 92:374-379

8. Kennedy NI, Wijdicks CA, Goldsmith MT, Michalski MP, Devitt BM, Arøen A et al (2013) Kinematic analysis of the posterior cruciate ligament, part 1: the individual and collective function of the anterolateral and posteromedial bundles. Am J Sports Med 41:2828-2838

9. Liddle AD, Judge A, Pandit H, Murray DW (2014) Adverse outcomes after total and unicompartmental knee replacement in 101 330 matched patients: a study of data from the National Joint Registry for England and Wales. Lancet 384:1437-1445

10. Logan M, Williams A, Lavelle J, Gedroyc W, Freeman M (2004) The effect of posterior cruciate ligament deficiency on knee kinematics. Am J Sports Med 32:1915-1992

11. Nakagawa $S$, Johal P, Pinskerova V, Komatsu T, Sosna A, Williams A et al (2004) The posterior cruciate ligament during flexion of the normal knee. J Bone Jt Surg Br 86:450-456

12. Nasab SH, List R, Oberhofer K, Fucentese SF, Snedeker JG, Taylor WR (2016) Loading patterns of the posterior cruciate ligament in the healthy knee: a systematic review. PLoS One 11:e0167106

13. No authors listed (2019) 16th Annual Report 2019. National Joint Registry for England, Wales, Northern Ireland and the Isle of Man

14. Okazaki K, Takayama Y, Osaki K, Matsuo Y, Mizu-uchi H, Hamai $S$ et al (2015) Subclinical cartilage degeneration in young athletes with posterior cruciate ligament injuries detected with T1 $\rho$ magnetic resonance imaging mapping. Knee Surg Sports Traumatol Arthrosc 23:3094-3100

15. Pandit H, Jenkins C, Gill H, Smith G, Price A, Dodd C et al (2011) Unnecessary contraindications for mobile-bearing unicompartmental knee replacement. J Bone Jt Surg Br 93:622-628

16. Wj Wang, Sun Mh, Palmer J, Liu F, Bottomley N, Jackson W et al (2018) Patterns of compartment involvement in end-stage knee osteoarthritis in a chinese orthopedic center: implications for implant choice. Orthop Surg 10:227-234

17. White S, Ludkowski P, Goodfellow J (1991) Anteromedial osteoarthritis of the knee. J Bone Jt Surg Br 73:582-586 
18. Willis-Owen CA, Brust K, Alsop H, Miraldo M, Cobb JP (2009) Unicondylar knee arthroplasty in the UK National Health Service: an analysis of candidacy, outcome and cost efficacy. Knee $16: 473-478$

19. Wilson HA, Middleton R, Abram SG, Smith S, Alvand A, Jackson WF et al (2019) Patient relevant outcomes of unicompartmental versus total knee replacement: systematic review and meta-analysis. BMJ 364:1352
Publisher's Note Springer Nature remains neutral with regard to jurisdictional claims in published maps and institutional affiliations. 